\title{
Magnetic anisotropy from single atoms to large monodomain islands of $\mathrm{Co} / \mathrm{Pt}(111)$
}

\author{
Pietro Gambardella, Stefano Rusponi, T. Cren, Nicolas Weiss, Harald Brune* \\ Institut de physique des nanostructures, École polytechnique federale de Lausanne, CH-1015 Lausanne, Switzerland
}

Available online 12 January 2005

Presented by Guy Laval

\begin{abstract}
By studying the magnetic behavior of self-assembled Co islands on a single-crystal metal surface, $\operatorname{Pt}(111)$, we show how the magnetic anisotropy evolves from isolated atoms to monolayer islands and films. Single Co adatoms are found to have a giant magnetocrystalline anisotropy energy of $E_{\mathrm{a}}=9.3 \pm 1.6 \mathrm{meV} /$ atom arising from the combination of partly preserved orbital moments $\left(m_{\mathrm{L}}=1.1 \mu_{\mathrm{B}}\right)$ and strong spin-orbit coupling induced by the Pt substrate. Combined scanning tunneling microscopy and X-ray magnetic circular dichroism experiments performed for differently sized small two-dimensional Co islands establish a clear connection between $E_{\mathrm{a}}$ and $m_{\mathrm{L}}$, both quantities decrease sharply with the lateral coordination of the magnetic atoms. In accordance with this, Kerr magnetometry experiments, again performed in conjunction with scanning tunneling microscopy, reveal that the anisotropy energy of large two-dimensional Co islands is almost entirely determined by the relatively low number of perimeter atoms, having $E_{\mathrm{a}}=0.9 \pm 0.1 \mathrm{meV} /$ atom. These results confirm theoretical predictions and are of fundamental value the understanding of how the magnetic anisotropy develops in finite-size magnetic particles. Identification of the role of perimeter and surface atoms opens up new opportunities for engineering the anisotropy and the moment of a magnetic nanostructure. To cite this article: P. Gambardella et al., C. R. Physique 6 (2005).
\end{abstract}

๑) 2004 Académie des sciences. Published by Elsevier SAS. All rights reserved.

\section{Résumé}

Anisotropie magnétique de l'atome isolé aux îlots monocouches de Co/Pt(111). En étudiant les propriétés magnétiques de particules de Co auto assemblées sur une surface d'orientation (111) d'un monocristal de Pt, nous montrons comment l'anisotropie magnétique évolue de l'atome isolé aux îlots monocouches et aux films ultraminces. Nous trouvons que les atomes de Co isolés adsorbés en surface ont une énergie d'anisotropie magnétocrystalline de $E_{\mathrm{a}}=9.3 \pm 1.6 \mathrm{meV} /$ atome provenant de la combinaison d'un moment orbital conservé $\left(m_{\mathrm{L}}=1.1 \mu_{\mathrm{B}}\right)$ et d'un fort couplage spin-orbite induit par le substrat de Pt. Des expériences combinées de microscopie à effet tunnel et de dichroïsme circulaire magnétique à rayons $\mathrm{X}$, effectués sur des îlots de Co de petit taille établissent une connexion claire entre $E_{\mathrm{a}}$ et $m_{\mathrm{L}}$, chacune de ces quantités décroissant rapidement avec la coordination latérale des atomes magnétiques. En conformité avec ceci, nous trouvons en employant la magnétométrie par effet Kerr et le microscope à effet tunnel que l'énergie d'anisotropie de grands îlots bidimensionnels de Co est presque entièrement déterminée par le nombre relativement faible d'atomes au périmètre, ayant $E_{\mathrm{a}}=0.9 \pm 0.1 \mathrm{meV} /$ atome. Ces résultats confirment les prédictions théoriques et sont d'un intérêt fondamental pour comprendre comment l'anisotropie magnétique se développe dans les particules magnétiques de taille finie. L'identification du rôle des atomes au périmètre et des atomes de surface ouvre de nouvelles opportunités pour l'ingénierie de l'anisotropie et du moment de nanostructures magnétiques. Pour citer cet article :P. Gambardella et al., C. R. Physique 6 (2005).

() 2004 Académie des sciences. Published by Elsevier SAS. All rights reserved.

\footnotetext{
* Corresponding author.

E-mail address: harald.brune@epfl.ch (H. Brune).
} 
Keywords: Magnetism; Magnetic Anisotropy; Nanostructures; STM; XMCD; MOKE

Mots-clés : Magnetisme; Anisotropie magnétique ; Nanostructures

\section{Introduction}

It is well known that the magnetic properties of transition metal particles depend on the coordination of the constituent atoms [1-4]. Small clusters deposited on metal surfaces are predicted to have spin $\left(m_{\mathrm{S}}\right)$ and orbital $\left(m_{\mathrm{L}}\right)$ magnetic moments in between those of bulk compounds and free atoms [5-7], and to exhibit strong magnetic anisotropy with characteristic energies $\left(E_{\mathrm{a}}\right)$ of the order of 1-10 $\mathrm{meV} /$ atom, i.e., a factor $10^{3}$ larger than bulk ferromagnetic metals [7,8]. In the case of single-domain particles, $E_{\mathrm{a}}$ determines the orientation and stability of the magnetization and is thus a crucial parameter for most applications of magnetic materials in modern technology. Van Vleck [9] was among the first to recognize that the orbital magnetization, via the spin-orbit interaction, connects the magnetocrystalline anisotropy to the atomic structure of magnetic materials.

In recent years, extensive work [10-19] on magnetic surfaces and thin films has shown that a lowering of the symmetry results in an increase of $m_{\mathrm{L}}$ compared to bulk systems, where the $d$-state hybridization and the crystal field effectively quench $m_{\mathrm{L}}$. This effect gives rise to a variety of interesting phenomena, such as enhanced and perpendicular magnetic anisotropy [2,12-16, 19]. Surface-supported nanoparticles offer additional degrees of freedom to tune the magnetic anisotropy by ad-hoc modifications of the particle size, shape, and coupling with the substrate, making nanometer sized systems attractive for basic investigations as well as for miniaturized data storage applications [20-22]. In this respect, self-assembled magnetic clusters grown on metal surfaces constitute an ideal system since their size, shape, and composition can be controlled with relative ease while producing them in numbers large enough for investigation with spatially integrating techniques [23,24]. In the following we present an extensive study of the evolution of the magnetic anisotropy energy in surface supported aggregates when their sizes evolve from one to about one thousand atoms. The data demonstrate a strong correlation between magnetic properties and morphological details. Reducing the atomic coordination number the anisotropy energy is seen to steeply increase and it assumes the giant value of $9 \mathrm{meV} /$ atom in the case of a single adatom. Identification and evaluation of the role of high and low coordinated magnetic atoms opens up new opportunities for material engineering which are likely to be of help in pushing forward the superparamagnetic limit in small magnetic particles.

\section{Orbital magnetic moment and magnetic anisotropy of single Co atoms and nanoparticles}

Gas-phase transition metal (TM) atoms possess large $m_{\mathrm{S}}$ and $m_{\mathrm{L}}$ according to Hund's rules, which are due to intra-atomic Coulomb interactions. In a solid, electron delocalization and crystal field effects compete with these interactions, causing a substantial decrease of $m_{\mathrm{S}}$ and partial or total quenching of $m_{\mathrm{L}}$. Theoretical calculations [7] predict such effects to be strongly reduced in TM impurities at non-magnetic surfaces owing to the decreased coordination, with implications also for the appearance of significant magnetic anisotropy (Fig. 1). TM clusters in the gas phase have also shown a strong dependence of the total magnetic moment $\left(m_{\mathrm{S}}+m_{\mathrm{L}}\right)$ on the particle size [3,25]. To date, however, fundamental points are still unclear. In particular, how does the magnetic anisotropy energy (MAE) evolve from single atoms to finite-sized magnetic particles, how does it correlate to the atomic magnetic moments, and how do both depend on the details of the atomic coordination. Such questions are

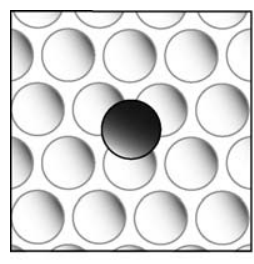

$+18 \mathrm{meV}$

$0.60(1.05) \mu_{\mathrm{B}}$

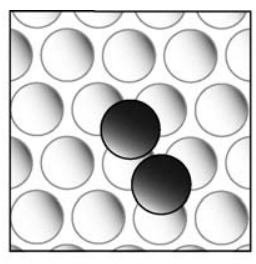

$$
+4.1 \mathrm{meV}
$$$$
0.38(0.74) \mu_{\mathrm{B}}
$$

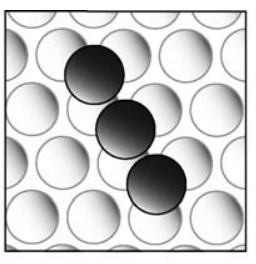

$$
+3.7 \mathrm{meV}
$$$$
0.34(0.67) \mu_{\mathrm{B}}
$$

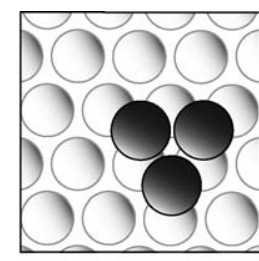

$+2.2 \mathrm{meV}$

$0.25 \mu_{\mathrm{B}}$

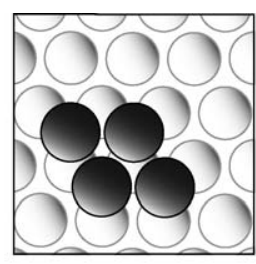

$$
\begin{gathered}
+0.75 \mathrm{meV} \\
0.22 \mu_{\mathrm{B}}
\end{gathered}
$$

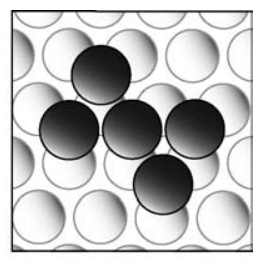

$+1.8 \mathrm{meV}$

$0.27 \mu_{\mathrm{B}}$

Fig. 1. Ab-initio calculation of $E_{\mathrm{a}}$ and $m_{\mathrm{L}}$ for Co on $\mathrm{Pt}(111)$ using the spin-polarized-relativistic Korringa-Kohn-Rostocker Green's function method in the spin-density approximation [30]. The Co atoms are on $f c c$ sites. The values of $m_{\mathrm{L}}$ between brackets have been computed within the orbital polarization scheme with a 50\% reduced Racah parameter. The reported values have been averaged over all Co sites for a given island, although site differences may well be relevant (see, e.g., [34]). 
(a)
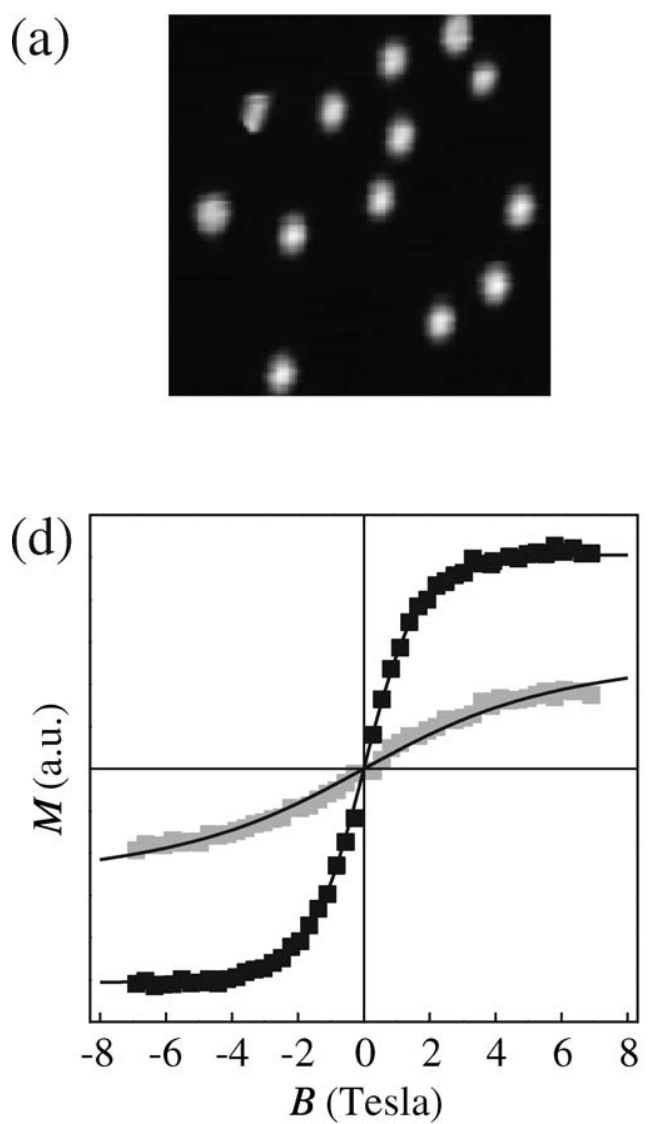

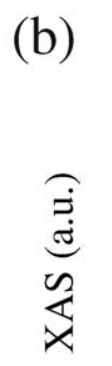

(c)
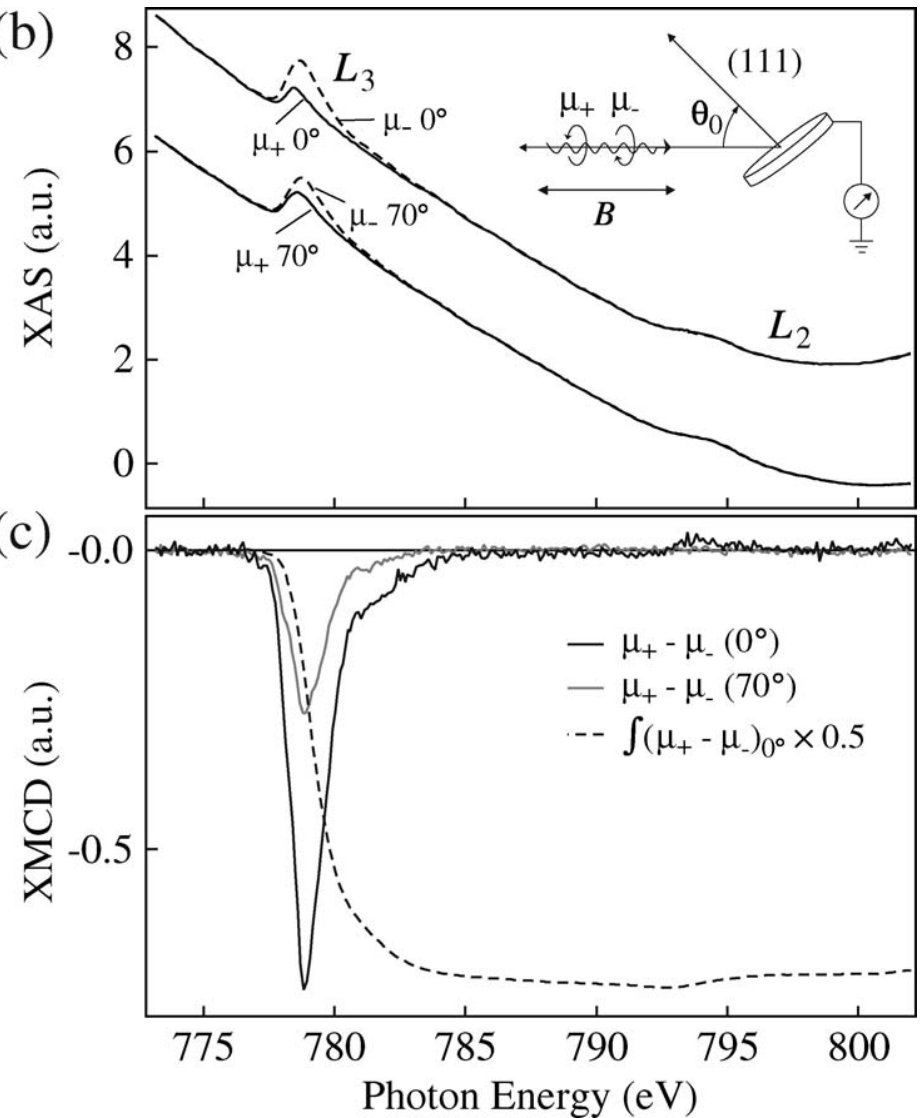

Fig. 2. (a) STM image of isolated Co adatoms on Pt(111) (coverage $0.010 \mathrm{ML}, 85 \AA \times 85 \AA$ ); (b) $L_{3,2}$ XAS spectra of isolated Co adatoms $(0.010 \mathrm{ML})$ at $T=5.5 \pm 0.5 \mathrm{~K}, B=7 \mathrm{~T}$ taken with parallel $\left(\mu_{+}\right)$and antiparallel $\left(\mu_{-}\right)$alignment of light helicity with respect to $B$ at $\theta_{0}=0^{\circ}$, $70^{\circ}$ relative to the surface normal, as shown in the inset. The spectra at $70^{\circ}$ have been normalized to the $\left(\mu_{+}+\mu_{-}\right) L_{3}$ intensity at $0^{\circ}$ in order to eliminate the dependence of the electron yield on the sample orientation; (c) XMCD spectra $\left(\mu_{+}-\mu_{-}\right)$obtained for the $\theta_{0}=0^{\circ}$ and $70^{\circ}$ magnetization directions. The dashed line is the integrated XMCD at $\theta_{0}=0^{\circ}$; (d) Magnetization curves at $\theta_{0}=0^{\circ}$ (filled symbols) and 70 (empty symbols) measured at $T=5.5 \mathrm{~K}$. The points represent the peak of the $L_{3} \mathrm{XMCD}$ intensity at $778.6 \mathrm{eV}$ divided by the pre-edge intensity at $775 \mathrm{eV}$ as a function of $B$. The difference between the $\theta_{0}=0^{\circ}$ and $70^{\circ}$ curves is consistent with the XAS-normalized XMCD spectra. The solid lines are fits to the data as described in [30].

not only relevant in bridging the gap between the atomic and solid-state descriptions of magnetic phenomena, but also because they provide guidelines for the engineering of nanostructures with novel or enhanced magnetic properties, as shown in the next section.

Here we report on the measurement of $m_{\mathrm{L}}$ and $E_{\mathrm{a}}$ in single surface adatoms and small, two-dimensional particles in the critical size range below one hundred atoms. To construct such small particles we have exploited the epitaxial growth of Co on $\mathrm{Pt}(111)$ in ultra-high-vacuum (UHV), which allows us to obtain a narrow size distribution by means of kinetically controlled growth [23]. This technique exploits the energetic hierarchy of the different diffusion and dissociation processes of adatoms at surfaces, and their dependence on the substrate symmetry. Control is achieved by means of external parameters such as, e.g., the substrate temperature and deposition flux, allowing atoms to diffuse and nucleate forming aggregates of defined size and shape [23]. The Co/Pt system was chosen in order to maximize the MAE effects, since hcp-Co presents the largest MAE among $3 d$ ferromagnetic elements $(0.045 \mathrm{meV}$ /atom compared to, e.g., $0.005 \mathrm{meV} /$ atom for $\mathrm{Fe})$. In addition, CoPt alloying in the bulk-ordered $\mathrm{L} 1_{0}$ phase results in a MAE increase up to $0.8 \mathrm{meV} / \mathrm{Co}$ atom [21], owing to the strong spin-orbit coupling of the Pt $5 d$ states.

Fig. 2(a) shows a scanning tunneling microscope (STM) image of isolated Co adatoms obtained by depositing 0.01 monolayers $\left(1 \mathrm{ML}=1.50 \times 10^{15}\right.$ atoms $\left./ \mathrm{cm}^{2}\right)$ at $T=5.5 \mathrm{~K}$, for which surface diffusion is completely inhibited. X-ray magnetic circular dichroism (XMCD) [26] measurements were performed to probe the adatom magnetism by detecting X-ray absorption spectra (XAS) at the Co $L_{2,3}$ edges ( $2 p$ to $3 d$ transitions) using left and right circularly polarized light in the total electron yield 
mode (Fig. 2(b)). The measurements were carried out on beamlines ID08 and ID12B at the European Synchrotron Radiation Facility, where the samples have been grown in-situ in UHV conditions. The XMCD signal (Fig. 2(c)), is the difference between the XAS spectra recorded for parallel $\left(\mu_{+}\right)$and antiparallel $\left(\mu_{-}\right)$alignment of the photon helicity with the applied field $B$. Fields of up to $7 \mathrm{~T}$ were used to magnetize the sample at angles $\theta_{0}=0^{\circ}, 70^{\circ}$ with respect to the surface normal. The adatom XAS spectra show relatively weak absorption features compared to the Pt background due to the extremely low concentration of Co adatoms. However, the XMCD signal is very large relative to the total Co absorption signal, indicating the presence of strong magnetic moments localized at the Co atoms. Two effects are characteristic of single adatom magnetic properties. First, the vanishing intensity of the XMCD at the $L_{2}$ edge indicates unusually strong orbital magnetism; second, the large difference between the magnetization measured in-plane and out-of-plane up to $7 \mathrm{~T}$ (Fig. 2(d)) reveals an extraordinary MAE.

According to the XMCD sum rules [27-29], we obtain $m_{\mathrm{L}}=1.1 \pm 0.1 \mu_{\mathrm{B}}$ /atom measured parallel to the easy axis direction $\left(\theta_{0}=0^{\circ}\right)$ [30]. This value is considerably reduced with respect to the $3 \mu_{\mathrm{B}}$ of a gas-phase Co atom, but extremely high for a metallic bulk system, where $m_{\mathrm{L}}$ is usually reduced to $\sim 0.1-0.2 \mu_{\mathrm{B}}$ owing to the hybridization of the $d$-states. The explanation lies with the reduced coordination of an isolated atom adsorbed on top of a flat surface, favoring $d$-electron localization and thus the survival of atomic-like character in the $3 d$ orbitals. The presence of such a large orbital magnetization has significant consequences for the magnetic anisotropy.

The MAE was determined by measuring the adatom magnetization parallel to $B$, with $B$ applied along different directions with respect to the surface normal, as shown in Fig. 2(d). A fit of the magnetization assuming classical, coherent reversal of the magnetization and uniaxial anisotropy yields $E_{\mathrm{a}}=9.3 \pm 1.6 \mathrm{meV} /$ atom [30], which is an exceptionally large value. Typical systems with high MAE are $\operatorname{SmCo}_{5}\left(E_{\mathrm{a}}=1.8 \mathrm{meV} / \mathrm{Co}\right.$ atom [21] $), \mathrm{Co} / \mathrm{Pt}, \mathrm{Co} / \mathrm{Au}$ multilayers $\left(E_{\mathrm{a}} \sim 0.3 \mathrm{meV} / \mathrm{Co}\right.$ atom $[12,14])$, and one-dimensional Co atomic chains $\left(E_{\mathrm{a}}=2.0 \mathrm{meV} / \mathrm{Co}\right.$ atom [19]). Different effects combine in establishing the magnitude of the MAE for the Co adatoms. First, the reduced coordination leads to $3 d$-electron localization (band narrowing), which augments the spin-orbit energy due to increases in the local density of states near the Fermi level and the orbital and spin magnetic moment [31,32]. Second, the strong spin-orbit coupling of the Pt $5 d$-states results in additional MAE of the induced magnetization, an effect common to CoPt compounds [33].

The predominant influence of the atomic coordination on the MAE and $m_{\mathrm{L}}$ is revealed by measurements on two-dimensional, monolayer particles obtained by statistical growth and annealing for average sizes $1<n \leqslant 5$ atoms, and by diffusion-controlled aggregation at $T<130 \mathrm{~K}$ for $5<n \leqslant 40$ atoms. Fig. 3 reports $m_{\mathrm{L}}$ and $E_{\mathrm{a}}$ as a function of $n$. The smaller $n$ is the larger the $m_{\mathrm{L}}, E_{\mathrm{a}}$ dependence on changes of the cluster size. Particles with $n=3$ and 4 atoms have $m_{\mathrm{L}}$ reduced to $0.78 \pm 0.05$ and $0.59 \pm 0.05 \mu_{\mathrm{B}}$ /atom, respectively. One-atom variations of the particle size cause significant reductions of $E_{\mathrm{a}}$ : for $n=3$ atoms, $E_{\mathrm{a}}=3.3 \pm 0.4 \mathrm{meV} /$ atom, only about $30 \%$ of the single adatom value. Ab-initio calculations (Fig. 1) [30,34] show that the atomic coordination rather than the absolute particle size is the key to determine $m_{\mathrm{L}}$ and $E_{\mathrm{a}}$ (see Fig. 1). In the experiment, the finite size distribution of the epitaxially grown Co particles does not strictly allow for such a distinction. However, the data reported in the next section show how the practical consequences of this effect can be exploited in much larger Co structures to enhance the total MAE. The comparison between Figs. 3(a) and 3(b) also demonstrates the correlation between $m_{\mathrm{L}}$ and $E_{\mathrm{a}}$, as expected from a perturbative treatment of the spin-orbit interaction. According to Bruno [31,32] and van der Laan [31,32] $E_{\mathrm{a}}$ is directly related to the anisotropy of $m_{\mathrm{L}}$, and (in TM atoms with more than half-filled $d$-electron shells) the easy axis is

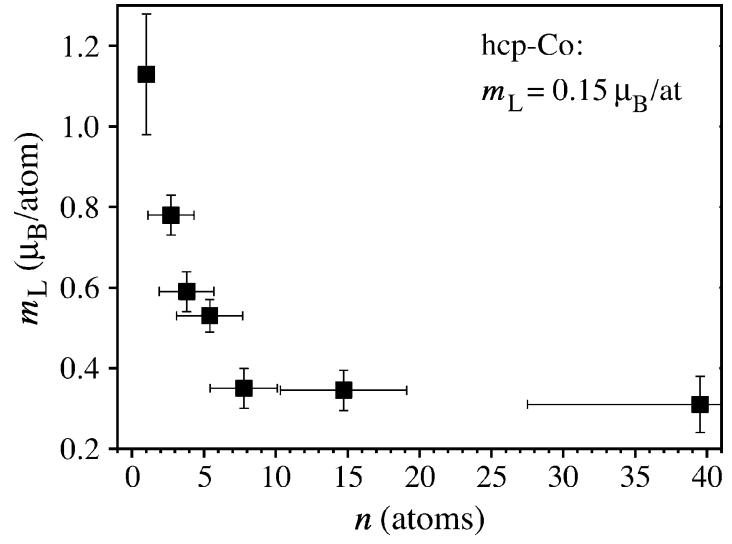

(a)

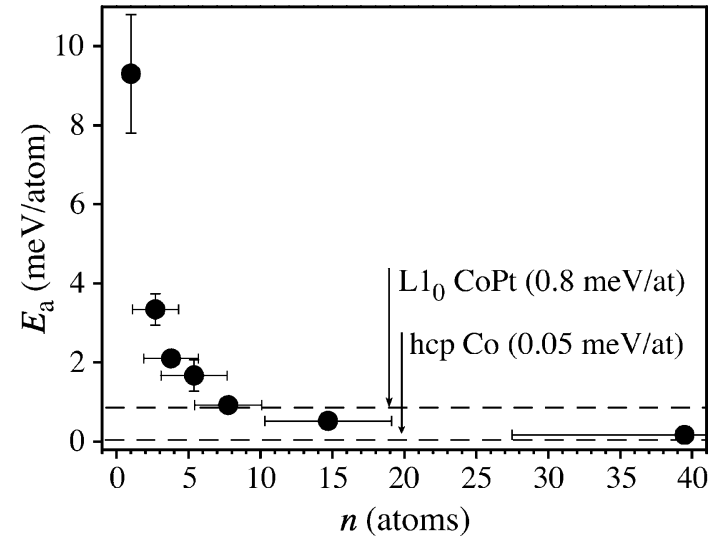

(b)

Fig. 3. (a) $m_{\mathrm{L}}$ and (b) $E_{\mathrm{a}}$ as a function of average island size $n$ for Co/Pt(111). For comparison, the dashed lines show $E_{\mathrm{a}}$ of the $\mathrm{L} 1_{0}$ CoPt alloy and hcp-Co, respectively. The error bars on the horizontal scale represent the standard deviation of the size distribution as determined by STM. From [30]. 
the direction where $m_{\mathrm{L}}$ is maximum. In our experiment, the incomplete saturation of the magnetization close to the hard axis direction (Fig. 2(d)), does not allow a precise estimate of the $m_{\mathrm{L}}$ anisotropy via the XMCD sum rule for the orbital moment. However, it is clear that a large $m_{\mathrm{L}}$ anisotropy, hence large $E_{\mathrm{a}}$ values, can be observed only if $m_{\mathrm{L}}$ itself is large, thus explaining the observed correlation between $E_{\mathrm{a}}$ and $m_{\mathrm{L}}$ reported in Fig. 3.

Finally, a theoretical exercise of some interest is the estimate of the MAE due to the induced polarization of the Pt substrate. If we imagine that $E_{\mathrm{a}}$ can be decomposed in partial contributions arising from Co and Pt sites (which is strictly not true since hybridization with Pt modifies also the character of the $d$-states localized on Co sites), the Pt substrate contributes to about $15 \%$ of the total MAE calculated for a single Co adatom. Because of the strong decrease of the Co MAE with increased coordination, however, the Pt share increases to $30 \%$ for the dimer and up to $60 \%$ for the pentamer, eventually providing the dominant MAE contribution in $\mathrm{Co} / \mathrm{Pt}$ multilayers [33].

\section{Temperature dependence of the zero field susceptibility of an ensemble of polydisperse 2D monodomain islands}

The results on 2D Co clusters containing less than a few tens of atoms suggest that the size of ferromagnetic particles with stable magnetization direction at room temperature can be made smaller than formerly anticipated, by artificially reducing the coordination of the magnetic atoms forming the particles. The industrial requirement for non-volatile data storage on hard disks is 10 years stability for a written bit, which implies an overall MAE of $K=1.2 \mathrm{eV}$ per magnetic grain of the recording medium. Assuming the Co-Co adatom coordination of 2, and a homogeneous magnetization, the data in the previous section imply a lower limit of about 400 atoms per grain. The ultimate limit will be to use a single grain to store one bit, which would mean 400 atoms per bit. Currently, one uses about $10^{3}$ grains per bit and each grain contains $10^{5}$ atoms. Whether single grain bits can be realized hangs on the homogeneity of the magnetic properties $M, K$, and of the direction of the easy axis. Note that similar issues are of relevance for the downscaling of the cells of magnetic random access memories (MRAMs).

A lower limit to the atomic coordination is set by the requirement that ferromagnetic order within the particle has to be established up to the temperatures of interest and up to a large inclination of $\mathbf{M}$ away from the easy axis appearing during magnetization reversal. Reducing the coordination reduces the ferromagnetic exchange energy. One illustration is one-dimensional chains, where the ferromagnetic correlation length is small and spin blocks with opposite magnetization form even at very low temperature [19,35]. Atoms in 2D or 3D nanostructures have higher mean coordination such that they may remain a ferromagnetically ordered unit up to high temperatures. One speaks of single domain particles which have homogeneous magnetization and are well described by a macrospin $M$ to which the aligned moments of the constituent atoms all add up.

In the remainder of this article we present results on 2D single domain particles in a size range of the order of 1000 atoms and we restrict ourselves to the system $\mathrm{Co} / \mathrm{Pt}(111)$ for the sake of comparison with the section above. The morphology of the island ensemble has again been characterized with STM and the zero-field susceptibility $\chi$ has been measured in-situ with surface magneto optical Kerr effect (MOKE). Under UHV conditions, there is no oxide shell and all constituent atoms are ferromagnetically ordered. Thus the particle morphology contributing to the magnetic signal is accessible at the atomic scale and a one-to-one correlation to the magnetic properties as a function of shape and size can be established. As we will show below this allows determination of the MAE of surface versus perimeter atoms, having a lateral coordination of 6 and 4 , respectively [36].

Before analyzing the experimental data we present an analytical model describing the transition from blocking to superparamagnetism of an ensemble of monodisperse, non-interacting monodomain particles. The Co islands on Pt(111) have uniaxial out-of-plane anisotropy and thus are characterized by two local minima for the potential energy $E$ as a function of the angle $\Theta$ between surface normal and magnetization $\mathbf{M}$. These minima correspond to the $u p$ and down orientation of $\mathbf{M}$ (see inset of Fig. 4). The anisotropy energy at zero field $K=E\left(90^{\circ}\right)$ is the energy of the transition state passed during magnetization reversal. The rate of barrier crossing for an ensemble of monodisperse particles, all having identical values of $K$ and $M$, or the time average of this rate for a single particle, is described in the case of small fields by an Arrhenius expression $v=v_{0} \exp \left((-K \pm H \cdot M) / k_{\mathrm{B}} T\right)$, with typically $v_{0}=1 \times 10^{10} \mathrm{~s}^{-1}$ [37] and $k_{\mathrm{B}}$ the Boltzmann constant. At zero field, the relaxation time $\tau$ is given by $\tau=1 / 2 v=\tau_{0} \exp \left(K / k_{\mathrm{B}} T\right)$, with $\tau_{0}=1 / 2 \nu_{0}$. The barrier is readily overcome if $T>T_{\mathrm{b}}=K / k_{\mathrm{B}} \ln \left(1 / \omega \tau_{0}\right)$, where $\omega$ is related to the observation time $t=2 \pi / \omega$. In our case $\omega$ is the sweep frequency of the external magnetic field used to measure $\chi . T_{\mathrm{b}}$ is the blocking temperature, defined by the temperature where the ensemble reaches half of its thermodynamic equilibrium susceptibility $\chi$ eq. For $T>T_{\mathrm{b}}$ the particles are superparamagnetic, and $\chi(T)=\chi_{\mathrm{eq}}(T)$. For $T<T_{\mathrm{b}}$ the particles are blocked in a fixed magnetization state (up or down), and hence $\chi(T)=0$. This is the state where information can be stored. In the vicinity of $T_{\mathrm{b}}$ the state of the system is determined by the kinetics of barrier crossing.

To fully capture the kinetics and thermodynamics we first calculated the zero-field susceptibility by numerically solving the master equations to obtain the occupation of the two energy minima, and by allowing for thermal fluctuations around these minima (see symbols in Fig. 4). Since we are interested in the limit of small fields, we can also linearize the master equations to 


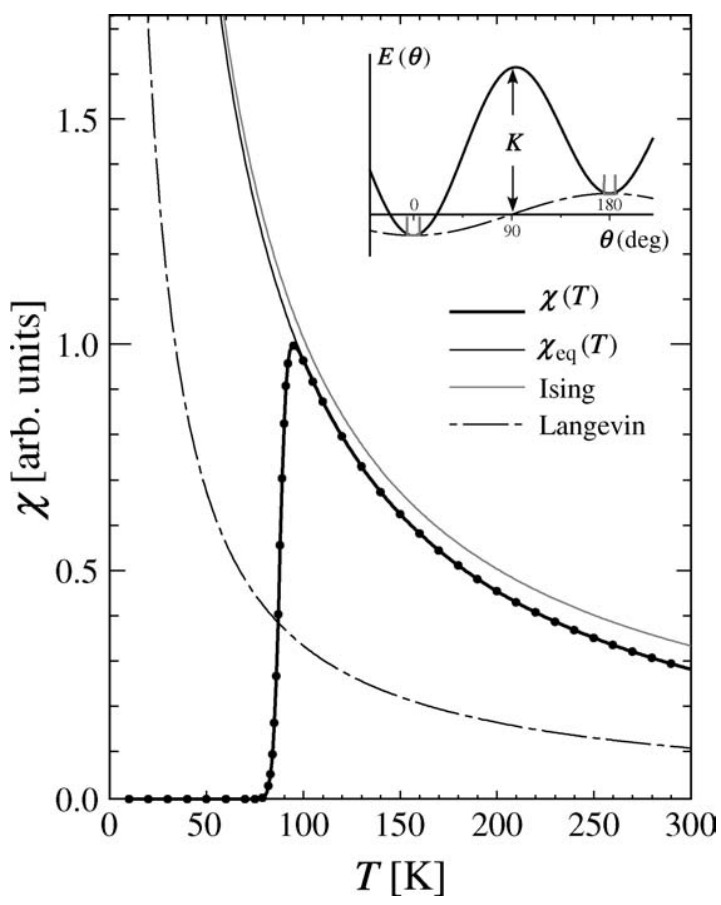

Fig. 4. Zero-field magnetic susceptibility $\chi(T)$ for an ensemble of monodisperse, uniaxial particles $(K=200 \mathrm{meV}, M=1500$ atoms $\times$ $2.1 \mu_{\mathrm{B}}=182 \mathrm{meV} / \mathrm{T}$, triangular field sweep with $\omega=0.3 \mathrm{~Hz}$ ). The analytical model (thick curve) perfectly describes the blocking to superparamagnetic transition as evidenced by comparison with the numerical calculation (symbols). The equilibrium zero-field susceptibility, $\chi$ eq, is shown as thin curve. Inset: Energy of an uniaxial monodomain particle as function of the orientation of $\mathbf{M}$ with respect to an out-of-plane external field $\mathbf{H}$ in the Ising model (2 states: up and down), in the Langevin model (continuum of states, no anisotropy, $E$ is given by Zeeman energy $\mathbf{H} \cdot \mathbf{M}$ ), and in the full model incorporating the anisotropy energy $K \cdot \chi(T)$ derived from the Ising (full gray curve) and Langevin (dash-dotted curve) models are shown in the main figure for comparison. From [36].

describe the kinetics [38], and multiply by the equilibrium susceptibility $\chi_{\mathrm{eq}}(T)$ to derive the following analytical expression for the real part of the complex zero-field susceptibility, which is the quantity we measure:

$$
\chi(T)=\frac{1}{1+\omega^{2} \tau^{2}} \chi \mathrm{eq} .
$$

$\chi_{\mathrm{eq}}(T)$ (see thin line in Fig. 4) has been calculated by Chantrell et al. [39], and given in the following form by Fruchart et al. [40]:

$$
\chi_{\mathrm{eq}}(T)=M^{2}\left[\frac{\exp \left(K / \mathrm{k}_{\mathrm{B}} T\right)}{\sqrt{\pi \mathrm{Kk}_{\mathrm{B}} T} \operatorname{Erfi}\left(\sqrt{K / \mathrm{k}_{\mathrm{B}} T}\right)}-\frac{1}{2 K}\right] .
$$

Fig. 4 shows that our analytical solution for $\chi(T)$ (thick line) perfectly reproduces the numerical one. Note that the numerical model takes into account the full shape of the $E(\Theta)$ curve, while the analytical one only contains the maximum energy $K$ via the relaxation time. The agreement between the two shows that the small field limit is justified.

Despite the fact that the particles are monodisperse, the transition from blocking to superparamagnetic takes place over a finite temperature window of $\Delta T=2 k_{\mathrm{B}} T_{\mathrm{b}}^{2} / K$ ( $8 \mathrm{~K}$ in our example). The maximum of $\chi$ is located just above $T_{\mathrm{b}}$ and its value is approximately $\chi_{\max }=M^{2} / k_{\mathrm{B}} T_{\mathrm{b}} \propto M^{2} / K$. For comparison, we also show the infinite anisotropy limit, leading to a two state system (Ising model, see gray full curves in main figure and inset), and the vanishing anisotropy limit, characterized by an occupation of all orientations of $\mathbf{M}$ (Langevin model, dash-dotted curves). With increasing temperature $\chi_{\text {eq }}$ goes from the first to the second limit and therefore it decays slightly steeper than the $1 / T$ behavior characterizing the two limiting cases. It is seen that the Ising model is a good approximation for $\chi(T)$ if $T_{\mathrm{b}}<T<2 T_{\mathrm{b}}$, while the Langevin model may only be used for very high temperatures. $\chi(T)$ of an ensemble of polydisperse particles is given by summing over the individual particles, each one represented by given values of $M$ and $K$ determined from their area and perimeter using different assumptions on the origin of $M$ and $K$. 


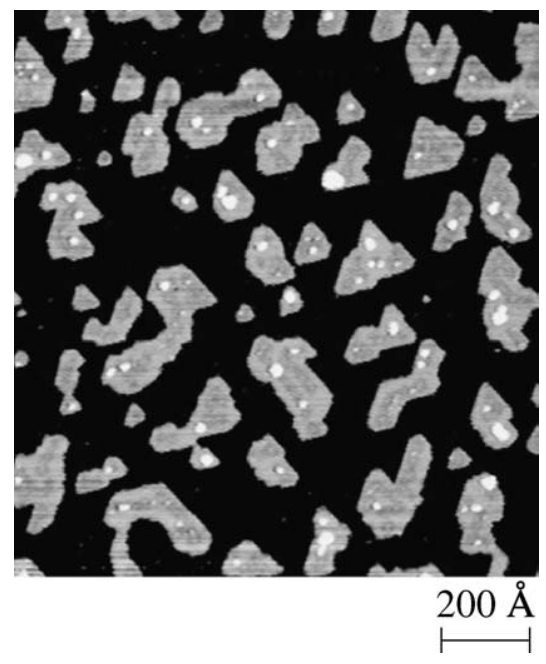

(a)

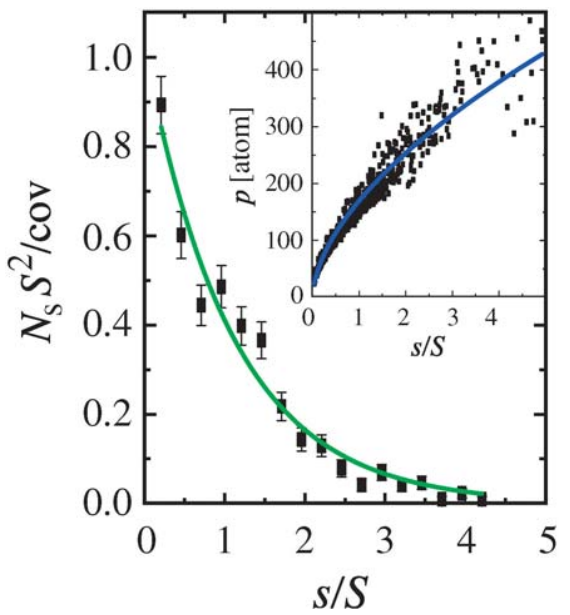

(b)

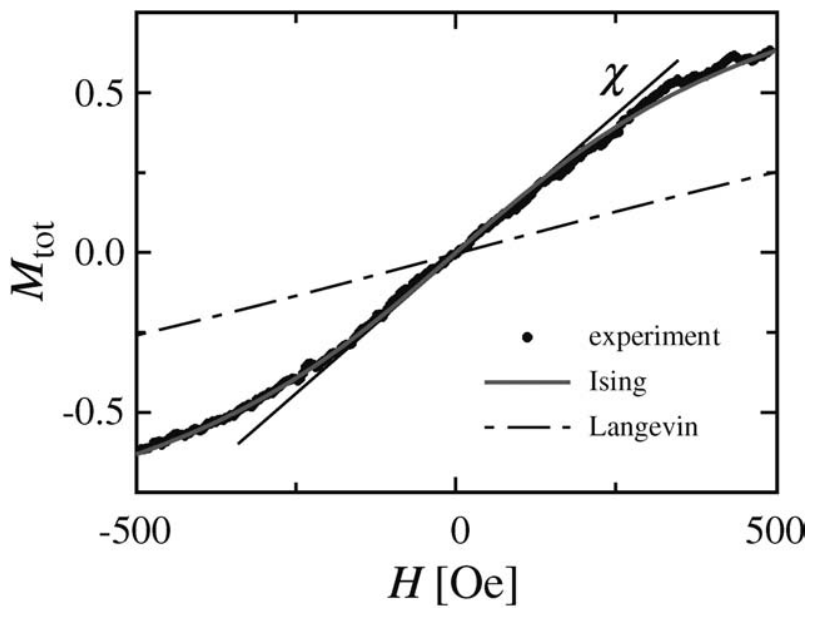

(c)

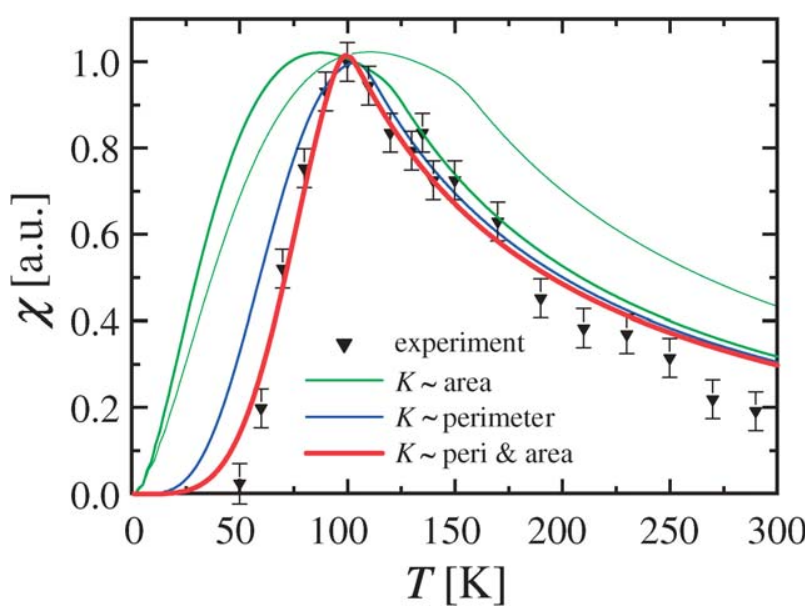

(d)

Fig. 5. Morphology and magnetism of an ensemble of compact one monolayer high Co islands on Pt(111): (a) STM image showing the morphology of islands (coverage $0.40 \mathrm{ML}$, mean island size $S=1200 \pm 1000$ atoms); (b) Size and perimeter length distribution. As customary in literature on epitaxial growth, this distribution has been normalized to yield unit area under the curve ( $s$ island size, $S$ average island size, $N_{\mathrm{S}}$ density of islands of size $s$, cov coverage in ML); (c) Magnetization as a function of out-of-plane field ( $T=150 \mathrm{~K}$, triangular field sweep with $\omega=2 \pi / 10 \mathrm{~s}$ ). The Ising fit is shown as blue line and yields $m=2.1 \mu_{\mathrm{B}}$ when taking the size distribution into account. The green line is the Langevin model with this value; (d) Temperature dependence of zero-field susceptibility $\chi(T)$ measured by MOKE. Curves are fits associating the same anisotropy energy $E_{\mathrm{a}}$ to all atoms per island (dark green $0.047 \mathrm{meV} /$ atom, light green $0.060 \mathrm{meV} /$ atom), solely to the perimeter atoms (blue $0.57 \mathrm{meV} /$ atom), and to both (red 0.9 and $-0.03 \mathrm{meV}$ ). From [36].

\section{Quantifying the contribution of perimeter vs. surface atoms to the magnetic anisotropy}

To elucidate the different role played by inner and edge atoms in a nanostructure we grew 2D islands with broad size and perimeter length distributions (Figs. 5(a), (b)). This allows us to probe a large range of both size and perimeter values with a single sample. The non-linear relationship between perimeter length and island area gives each of the two distributions its characteristic shape, thus enabling to disentangle the roles of the two atomic species.

For the determination of the magnetic moment $m$ per atom we measure with MOKE the magnetization as function of applied field at $T$ placing ourselves just above $T_{\mathrm{b}}$. The data are displayed as $M_{\mathrm{tot}}(H)$ in Fig. 5® and show the typical superparamagnetic reversible $S$-shaped curve. The magnetization reaches $60 \%$ of its saturation value at our experimentally available field of \pm 500 Oe. The vertical scale in Fig. 5๑ is calibrated by comparing with the signal from a full monolayer, which we can saturate, and by assuming the Kerr signal to be linear with coverage, $M_{\mathrm{sat}}(0.4 \mathrm{ML})=0.40 \times M_{\mathrm{sat}}(1.0 \mathrm{ML})$. For calculating the islands 
macrospins we assume that each atom has the same total magnetic moment $m$, thus the moment of an island containing $s$ atoms is given by $M=s m$. This assumption is justified since $m$ varies by much less than $20 \%$ for the size range of interest [41]. We saw above that the Ising model is a good approximation just above $T_{\mathrm{b}}$. Applying this model to fit the experimental magnetization curve and taking the size distribution into account leads to the blue curve yielding $m=2.1 \pm 0.2 \mu_{\mathrm{B}} /$ atom. This value is in good agreement with that expected from taking the sum of the calculated Co spin moment $m_{\mathrm{Co}, \mathrm{S}}=1.8 \mu_{\mathrm{B}}$ [42], its measured orbital moment $m_{\mathrm{Co}, \mathrm{L}}=0.2 \mu_{\mathrm{B}}$ [14], and the measured polarization of $\mathrm{Pt}$ at its interface with Co, amounting to $m_{\mathrm{Pt}}=0.2 \mu_{\mathrm{B}}$ [43]. When the size distribution is unknown, one typically represents the real system by an ensemble of monodisperse particles, all having the average size. We emphasize that this generally yields erroneous results; here it would give $m=3.0 \pm 0.2 \mu_{\mathrm{B}} /$ atom. In addition, using the same assumption and the Langevin model, would yield an unrealistic $m=6 \pm 1 \mu_{\mathrm{B}} /$ atom.

The MAE was determined by measuring the susceptibility at zero field $\chi$ (the indicated slope in Fig. 5(c)) as a function of temperature (Fig. $5(\mathrm{~d})$ ),$\chi(T)$ shows a broad peak around $100 \mathrm{~K}$ revealing a broad transition from blocking to superparamagnetic. This peak is followed by a roughly $1 / T$ decrease. The full curves are inferred from using the size and perimeter distributions as input to calculate $\chi(T)$ for the ensemble of particles from our analytical expression with different assumptions on the origin of the anisotropy per island, $K$. For the atomic moments we used in our fits $m=2.1 \mu_{\mathrm{B}} /$ atom, as determined above. Note, however, that the value of $m$ is irrelevant for the fit, as it only affects the scaling factor of the vertical scale in the $\chi(T)$ plot and the systematic uncertainty in $m$ coming from the fact that we cannot saturate our sample is irrelevant to the following conclusions on the origin and values of $K$. We further note that we attribute all the MAE to the Co atoms, and therefore the values we give include, as in the previous section, the effect of the polarized substrate.

First, we assume that each atom contributes the same amount, $K=s E_{\mathrm{a}}$, with $E_{\mathrm{a}}$ denoting the anisotropy per surface Co atom (green curves). Second, we assume that only the perimeter atoms contribute to the anisotropy, $K=p E_{\mathrm{a}}$, with $p$ the perimeter length in atoms (blue curve). Finally, we assume that surface atoms contribute the amount $E_{\text {as }}$, and perimeter atoms $E_{\text {ap }}$, thus $K=s E_{\text {as }}+p E_{\text {ap }}$ (red curve). The only free parameter of the fits is the anisotropy $E_{\text {a }}$ attributed to the surface, or perimeter atoms, or to both. Because $\chi(T)$ is dominated by terms containing $K / T$, a variation of $E_{\mathrm{a}}$ stretches or compresses the curves along the $x$-axis, while keeping their origin fixed (see dark and light green curves in Fig. 5(d). However, there is no way to change their overall shape which is controlled by the size and perimeter distributions.

From inspection of Fig. 5(d) it is obvious that attributing the anisotropy of the nanostructures entirely to the surface or interface fails because the green curve is far too broad. In addition, the inferred value of $E_{\mathrm{a}}=0.047 \pm 0.005 \mathrm{meV} / \mathrm{atom}$ is comparable to Co bulk, making difficult any reconciliation with typical thin film values of $0.1-0.2 \mathrm{meV} /$ atom [14,44,45]. The blue curve fits our data much better and associates $E_{\mathrm{a}}=0.57 \pm 0.05 \mathrm{meV}$ to the perimeter atoms and zero contribution to the surface atoms in the interior of the islands. Finally, the best fit is obtained when we take both inner and edge atoms into account and associate $E_{\mathrm{ap}}=0.9 \pm 0.1 \mathrm{meV}$ to the perimeter and $E_{\text {as }}=-0.03 \pm 0.01 \mathrm{meV}$ to the surface atoms. We note that the value $E_{\text {ap }}=0.9 \pm 0.1 \mathrm{meV}$ associated to the step atoms, having on average four in-plane neighbors, compares well to the MAE/atom measured by means of XMCD in small clusters with sizes of about 10 atoms, where the lateral coordination is comparable. Recently, $E_{\mathrm{a}}$ values of the same order of magnitude have also been reported for Co atoms forming monatomic chains attached to the $\{111\}$-facetted steps of a $\operatorname{Pt}(997)$ surface [19]. It is interesting to decompose $E_{\text {as }}$ into its magnetocrystalline and shape contributions, $E_{\text {as }}=E_{\text {cryst }}+E_{\text {shape }}$. We note that a variation of the demagnetization factor with island size is expected, but this transition occurs for much smaller islands than the one studied here [46], thus we assume a constant demagnetization factor of 1 and calculate a demagnetizing energy of $E_{\text {shape }}=-0.090 \mathrm{meV} /$ atom using the saturation magnetization of bulk Co. We get $E_{\text {cryst }}=0.06 \pm 0.01 \mathrm{meV} /$ atom which is more than counterbalanced by $E_{\text {shape }}$. This implies that without the anisotropy of the perimeter atoms the islands would be magnetized in-plane.

In the previous discussion we tacitly assumed the absence of magnetic interactions between the particles, possibly mediated by their dipolar stray field. One indication that such interactions are indeed absent comes from the agreement between experimental and calculated data for $\chi(T)$ in the superparamagnetic regime, where we observe the predicted slightly steeper than $1 / T$ decrease, while calculations show that interactions would considerably flatten this decrease [47]. On the other hand, the absence of interactions can also be rationalized by comparing the island switching field $H_{\mathrm{sw}}$, the applied field $H$, and the stray field $H_{\text {dipol }}$, created by all other islands at the location of one island. Assuming an ensemble of monodisperse particles with $M=s m=146 \mathrm{meV} / \mathrm{T}$ and $K=117 \mathrm{meV}$, and assuming that all of them are ferromagnetically aligned we get $H_{\text {dipol }}=100 \mathrm{Oe}$ (Eq. (4) of [48]). This value is below the applied field amplitudes, and it is an upper bound to the real dipolar field since the probability of all the neighbors of a given island being aligned is low. The temperature dependence of the switching field is approximately given by $[49,50]$

$$
H_{\mathrm{sW}}(T) \approx H_{0}\left(1-5 \sqrt{\frac{\mathrm{k}_{\mathrm{B}} T}{K}}\right) \approx H_{0}\left(1-\sqrt{\frac{T}{T_{\mathrm{b}}}}\right),
$$

where $H_{0}=2 \mathrm{~K} / \mathrm{M}=1.6 \times 10^{4} \mathrm{Oe}$. The switching field becomes comparable to the dipolar one only in the interval $0.99 T_{\mathrm{b}}<$ $T<T_{\mathrm{b}}$. In a polydisperse sample a small island may be superparamagnetic while the larger ones in is vicinity remain blocked. 
In this case the dipolar field simply adds to the external one which has a larger amplitude in our case such that we don't expect dipolar interactions. This qualitative argument signifies that much higher densities of non-interacting particles may be realized for out-of-plane magnetization than the density investigated here, which amounts to 2 Tera-particles/in ${ }^{2}$.

Our data highlight two important points. First the high magnetic anisotropy of low coordinated atoms, and, second the magnetocrystalline anisotropy of highly coordinated atoms that can eventually be compensated by shape anisotropy in perpendicular media. The first point rationalizes several results on the magnetic anisotropy of thin films reported in literature. The first indication of the role played by step atoms came from a couple of papers comparing the magnetism of Fe films on W(110) with smooth and high step density surfaces [51,52]. For the step atoms, the authors measured anisotropy energies twice as large as those of surface atoms and rationalized the effect by taking into account the broken symmetry at step sites in a Néel anisotropy model. Further indications came from Co films epitaxially grown on $\mathrm{Cu}(100)$. Weber et al. observed the Co magnetic anisotropy to oscillate as a function of the Co thickness. The period was exactly equal to one monolayer, corresponding to the variations of the film morphology alternating between filled and incompletely filled atomic layers [53]. On the same system was also observed that step decoration with minute amount of $\mathrm{Cu}$ induces a $90^{\circ}$ in-plane rotation of the easy magnetization axis of a 10 to 20 monolayers thick Co film [54,55]. The linear dependence of the anisotropy energy with the step density observed for fcc Co films grown on vicinal $\mathrm{Cu}(100)$ surfaces again indicates the step induced anisotropy the origin of which being attributed to the missing bond at the step edges while biaxial strain due to the in-plane lattice misfit was excluded [56,57]. More recently, XMCD measurements revealed a systematic enhancement of the anisotropy energy per atom with reduced size for Co [18] and $\mathrm{Fe}$ [58] clusters on $\mathrm{Au}(111)$, although no quantitative assignment of $E_{\mathrm{a}}$ values to core and perimeter atoms could be achieved.

The second point can be at first sight surprising because it contradicts several results on $\mathrm{Co} / \mathrm{Pt}(111)[43,44,59-62]$ and on multilayered $\mathrm{Co} / \mathrm{Pt}[14,45,63]$ and $\mathrm{Co} / \mathrm{Pd}$ [64-66] films all showing out-of-plane anisotropy. However, three theoretical works show the ideal Co monolayer on $\operatorname{Pt}(111)$ [42,67] and $\operatorname{Pd}(111)$ [68] to have in-plane anisotropy, the later result having been experimentally proofed [69]. In particular, Wu et al. [42], using a model with only one Pt layer and ignooring the internalcurrent contribution to the effective magnetic field [70], calculated a surface anisotropy of $E_{\text {cryst }}=45 \mu \mathrm{eV} /$ atom, very close to our estimation of $E_{\text {cryst }}=0.06 \pm 0.01 \mathrm{meV} /$ atom. The perpendicular anisotropy often observed in thin Co films can probably be ascribed to the non-homogeneity of the deposited films. Lundgreen et al. [71] observed 3D growth for Co deposited onto $\mathrm{Pt}(111)$ at room temperature. The 1.2 ML film shows a large fraction of the surface covered by the second layer. Moreover, room temperature growth produces site exchange between interfacial Co and Pt atoms, as demonstrated by STM images [71] and $\mathrm{X}$-ray magnetic diffraction [60]. Roughness increases the number of low coordinated sites and interfacial exchange increases the number of Co-Pt bonds. Both effects enhance the perpendicular anisotropy with respect to the ideal case of a perfect Co monolayer with an abrupt interface and no steps. A similar increase of the perpendicular anisotropy is produced by capping layers because polarization can be induced at the new interface $[69,72,73]$. For example it has been demonstrated that the inplane magnetization of $6 \mathrm{ML} \mathrm{Co/Pd(111)} \mathrm{turns} \mathrm{to} \mathrm{be} \mathrm{out-of-plane} \mathrm{after} \mathrm{capping} \mathrm{it} \mathrm{with} \mathrm{about} 2 \mathrm{ML}$ of $\mathrm{Cu}$ [65]. All these effects are absent in our sample since the islands were grown at low temperature and then shortly annealed in order to prevent exchange mechanisms, the islands are flat and they are observed in situ without the use of capping layers.

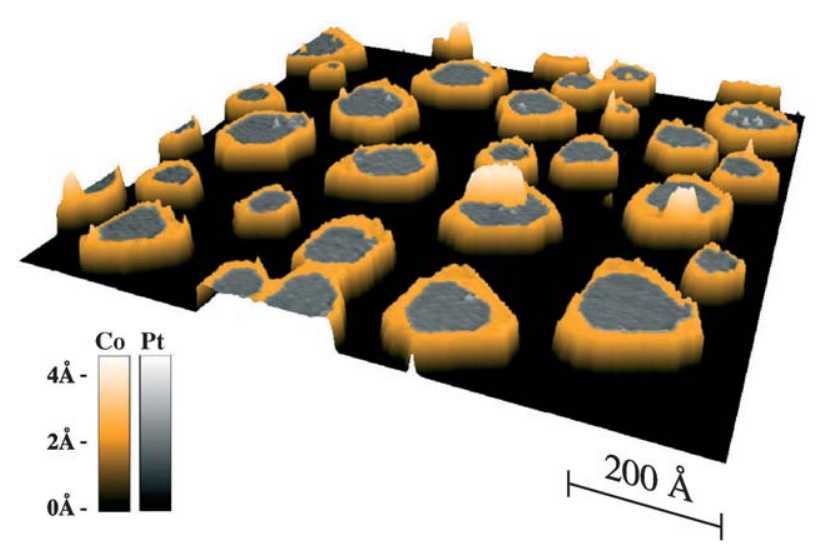

(a)

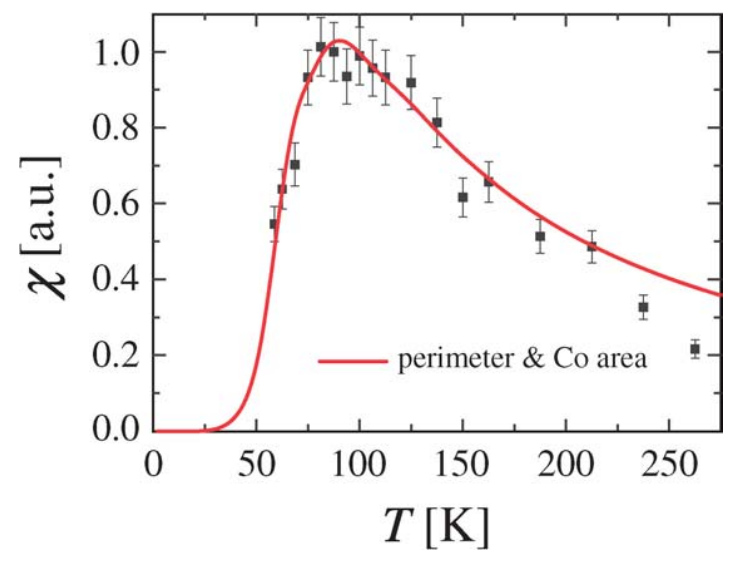

(b)

Fig. 6. Tailoring magnetic properties in bi-metallic islands: (a) 3D view of an STM image of one monolayer high islands with Pt core and an approximately 3 atom wide Co shell (deposition of $0.2 \mathrm{ML} \mathrm{Pt}$ at $130 \mathrm{~K}$ and annealing to $760 \mathrm{~K}$ generates the quasi hexagonal non-magnetic core; subsequent deposition of 0.2 ML Co at $220 \mathrm{~K}$ creates the Co rim). In the STM topographs, Co can be discerned from Pt by its $0.3 \AA$ larger apparent height which was used as color code; (b) $\chi(T)$ reveals that the Co-rim-Pt-core islands have the same anisotropy as the pure Co islands with equal perimeter length. From [36]. 
Our finding opens new possibilities to separately tune the anisotropy and moment of nanostructures. To exemplify this we produced bi-metallic one monolayer high islands with a nonmagnetic core ( $\mathrm{Pt}$ ) surrounded by a 2-3 atoms-wide magnetic rim (Co), see Fig. 6. We took care that the rim is at least two atoms wide in order to increase the exchange-coupling energy and thus enforce long-range ferromagnetic order (in monatomic Co chains, where the chain atoms have only two magnetic neighbors, ferromagnetic order has been reported to extend only over 15 atoms at $45 \mathrm{~K}$ [19]). It is seen from Fig. 6(b) that keeping the anisotropy energy per perimeter atom fixed to the value inferred above for the pure Co islands perfectly reproduces the switching behavior. The bi-metallic islands have identical anisotropy as their equally shaped pure Co counterparts; however, they have a much smaller overall moment due to their non-magnetic core.

\section{Enhanced zero-field susceptibility by partial oxidation}

Due to their reduced dimensions, small metallic magnetic particles created by metal epitaxy at surfaces are very sensitive to pollution, in particular to oxidation. The effect of oxidation on the magnetic properties can be quite complex. Since oxidation generally starts at the low coordinated sites, partial oxidation may provide helpful insight into the origin of magnetic properties, such as magnetic anisotropy. Generally, oxidation degrades the magnetic properties, for instance it has been reported to reduce the anisotropy [74]. However, in particular systems it may dramatically increase the anisotropy, e.g., due to exchange coupling with an antiferromagnetic $\mathrm{CoO}$ shell [75]. In this section we discuss the evolution of the magnetic properties of the 2D cobalt islands shown in Fig. 5 as function of their progressing oxidation.

STM images show clearly that the oxidation process begins at the island edge [76]. With increasing oxygen exposure, the dislocation pattern characteristic of clean Co is progressively replaced by a superstructure with approximately $(3 \times 3)$ unit cell and a periodicity of $10 \pm 1 \AA$. An oxygen dose of only 0.3 Langmuir is sufficient for the superstructure to cover the entire surface of small islands, whereas clean Co patches remain only in the center of the largest islands (Fig. 7).

MOKE measurements show that partial oxidation modifies the temperature dependence of $\chi$ in two ways. The temperature at which the maximum occurs is reduced, and the maximum taken on by $\chi(T)$ increases (Fig. 7(b)).

This apparently strange effect can be understood with the help of a qualitative argument. We know from the previous sections that the island magnetic anisotropy energy $K$ depends strongly on the perimeter atoms. Thus, for a very small amount of oxygen, for which only the island edge is oxygen covered due to the selective adsorption at step sites [77], the anisotropy drops strongly. The island magnetic moment is less sensitive to oxidation and decreases proportionally to the oxidized fraction of the island. In

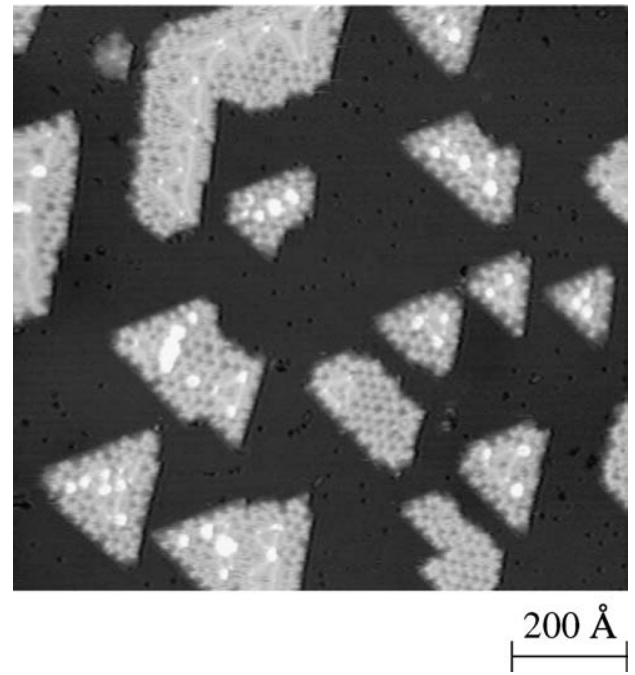

(a)

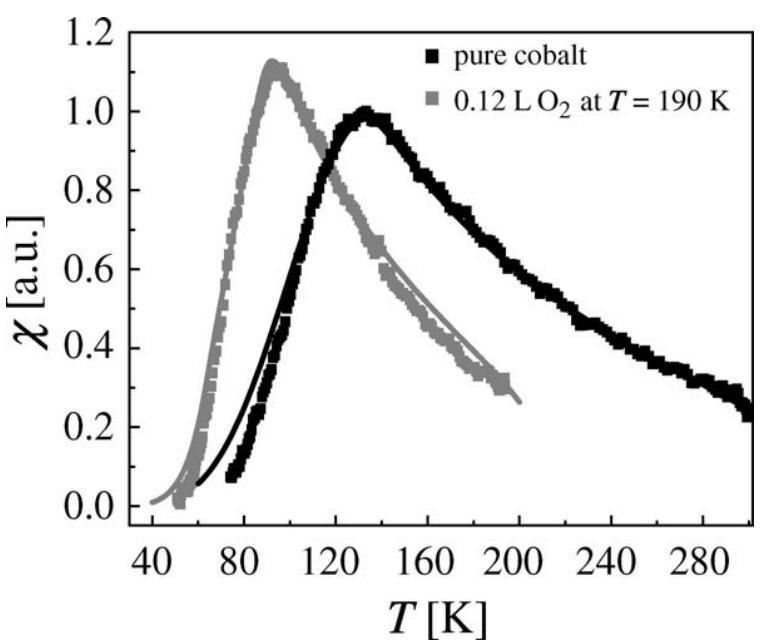

(b)

Fig. 7. Enhancement of the zero field susceptibility by oxygen adsorption: (a) STM image of Co islands after the exposure to $0.3 \mathrm{LO}{ }_{2}$. Only a small fraction of the larger islands have still the native strain relief pattern in their center, the remaining Co covered surface is transformed into an oxygen induced $(3 \times 3)$ structure; (b) In-phase zero field susceptibility $\chi$ as function of temperature for pure and partly oxygen covered Cobalt islands. Oxygen adsorption leads to a decrease of the blocking temperature and to an increase of $\chi_{\max }$. From [76]. 
a first approximation, we can suppose that $K$ decreases, i.e. $K_{\mathrm{Ox}}<K_{\mathrm{cl}}$, while the macrospin stays constant, i.e., $M_{\mathrm{cl}}=M_{\mathrm{OX}}$. Then, we obtain

$$
\frac{\chi_{\mathrm{ox}}^{\max }}{\chi_{\mathrm{cl}}^{\max }} \approx \frac{K_{\mathrm{cl}}}{K_{\mathrm{ox}}}>1 .
$$

With a more quantitative approach, the macrospin of an oxidized island can be written as $M(x)=(1-x) M_{\mathrm{cl}}$, where $x$ is the oxidized fraction of the island. We assume that for a given exposure, all the islands have the same oxidized fraction due to the fact that the oxygen capture rate is almost proportional to the island area. The surface anisotropy is affected in the same way as the macrospin, therefore we take $E_{\mathrm{S}}(x)=(1-x) s E_{\mathrm{as}}$. For the perimeter anisotropy, we consider that the oxidation begins at edges until the edges are completely saturated, then the oxidation progresses toward the centre of the islands. If the edge is not fully saturated, the number of edge atoms contaminated is simply given by $p_{\mathrm{ox}}=s x(s x<p)$, whereas if the edge is completely oxidized $(s x \geqslant p) p_{\mathrm{ox}}=p$, where $s$ and $p$ refer to the number of surface and perimeter atoms in the clean island. Then a straightforward expression for the perimeter anisotropy is $E_{\mathrm{p}}(x)=\left(p-p_{\mathrm{ox}}\right) E_{\mathrm{ap}}+p_{\mathrm{oxy}} E_{\mathrm{ap}}^{\mathrm{ox}}$ for the unsaturated case, and for the saturated one $E_{\mathrm{p}}(x)=p E_{\mathrm{ap}}^{\mathrm{ox}}$, where $E_{\mathrm{ap}}^{\mathrm{ox}}$ stands for the anisotropy of the edge after oxidation. For the clean parameters we keep the same $E_{\text {as }}$ and $E_{\text {ap }}$ determined above. The best fit to the data in Fig. 7(b) is obtained with $E_{\text {ap }}^{\text {ox }}=0.72 \mathrm{meV} /$ atom and $x=0.11$. The fact that $E_{\text {ap }}^{\mathrm{ox}}$ is only $21 \%$ lower than $E_{\text {ap }}$ seems surprising at first sight, since it signifies that the contamination of Co edge atoms lowers their anisotropy only by a relatively small amount. However, it is likely that the oxygen-saturated step atoms present a much lower magnetic anisotropy energy than the value deduced here, and that the new interface between the oxygen covered edge atoms and the clean Co atoms situated one row behind may augment their anisotropy far beyond the former surface value. Since the anisotropy is the sum of a surface and perimeter term, its reduction when all the perimeter atoms are contaminated can be higher than $21 \%$. With our set of parameters, we find that the anisotropy of an island of 500 atoms has decreased by $12 \%$, while the anisotropy of an island of 5000 atoms has decreased by $30 \%$. This clearly shows that in our model the anisotropy of the larger islands decreases much more rapidly than the anisotropy of the smaller ones, causing the observed compression of the MAE distribution [76].

\section{Conclusions}

We showed how the magnetic anisotropy energy of Co islands grown on $\mathrm{Pt}(111)$ by self-assembly evolves as their size increases from one to a few thousands atoms. In the limit of single adatoms we observed a MAE of 9 meV/atom, more than two orders of magnitude higher with respect to the bulk or thin film values. Due to the reduced atomic coordination the atoms recover part of their gas phase properties, e.g., their orbital moment is significantly larger than in bulk. The unquenched orbital magnetization is accompanied by a significant anisotropy of the orbital moment itself, which in turn induces a strong magnetic anisotropy owing to spin-orbit coupling. As a consequence of the strong coordination effect, the anisotropy of two-dimensional Co islands grown on $\mathrm{Pt}(111)$ is principally determined by the edge atoms. On the other hand, the overall magnetic moment of an island is determined by all atoms approximately to equal parts because the spin moment is less sensitive to the coordination and much larger than the orbital moment. This opens a new approach to fine-tune magnetic anisotropy and magnetic moment by adjusting the perimeter-to-surface ratio in magnetic nanostructures. Step atoms not only carry most of the magnetic anisotropy but they are also more reactive. Therefore partial oxidation quenches first the anisotropy leading to an increase of zero field susceptibility accompanied by a compression of the MAE distribution.

\section{References}

[1] J. Kanamori, in: G.T. Rado, H. Suhl (Eds.), Magnetism, vol. 1, Academic Press, New York, 1963.

[2] U. Gradmann, in: K.H.J. Buschow (Ed.), Handbook of Magnetic Materials, vol. 7, Elsevier, Amsterdam, 1993.

[3] I.M.L. Billas, A. Châtelain, W.A. de Heer, Science 265 (1994) 1682.

[4] G.M. Pastor, J. Dorantes-Dávila, S. Pick, H. Dreysse, Phys. Rev. Lett. 75 (1995) 326.

[5] K. Wildberger, V.S. Stepanyuk, P. Lang, R. Zeller, P.H. Dederichs, Phys. Rev. Lett. 75 (1995) 509.

[6] V.S. Stepanyuk, W. Hergert, K. Wildberger, R. Zeller, P.H. Dederichs, Phys. Rev. B 53 (1996) 2121.

[7] B. Nonas, I. Cabria, R. Zeller, P.H. Dederichs, T. Huhne, H. Ebert, Phys. Rev. Lett. 86 (2001) 2146.

[8] R. Félix-Medina, R. Guirado-Lòpez, J. Dorantes-Dávila, G.M. Pastor, J. Appl. Phys. 87 (2000) 4894.

[9] J.H. Van Vleck, Phys. Rev. 52 (1937) 1178.

[10] Y. Wu, J. Stöhr, B.D. Hermsmeier, M.G. Samant, D. Weller, Phys. Rev. Lett. 69 (1992) 2307.

[11] M. Tischer, O. Hjortstam, D. Arvanitis, J. Hunter Dunn, F. May, K. Baberschke, J. Trygg, J.M. Wills, B. Johansson, O. Eriksson, Phys. Rev. Lett. 75 (1995) 1602.

[12] D. Weller, J. Stöhr, R. Nakajima, A. Carl, M.G. Samant, C. Chappert, R. Mégy, P. Beauvillain, P. Veillet, G.A. Held, Phys. Rev. Lett. 75 (1995) 3752. 
[13] H.A. Dürr, G. van der Laan, B.T. Thole, Phys. Rev. Lett. 76 (1996) 3464.

[14] N. Nakajima, T. Koide, T. Shidara, H. Miyauchi, H. Fukutani, A. Fujimori, K. Iio, T. Katayama, M. Nyvlt, Y. Suzuki, Phys. Rev. Lett. 81 (1998) 5229.

[15] A.N. Anisimov, M. Farle, P. Poulopoulos, W. Platow, K. Baberschke, P. Isberg, R. Wäppling, A.M.N. Niklasson, O. Eriksson, Phys. Rev. Lett. 82 (1999) 2390-2393.

[16] F. Wilhelm, P. Poulopoulos, P. Srivastava, H. Wende, M. Farle, K. Baberschke, M. Angelakeris, N.K. Flevaris, W. Grange, J.-P. Kappler, G. Ghiringhelli, N.B. Brookes, Phys. Rev. B 61 (2000) 8647-8650.

[17] K.W. Edmonds, C. Binns, S.H. Baker, S.C. Thornton, C. Norris, J.B. Goedkoop, M. Finazzi, N.B. Brookes, Phys. Rev. B 60 (1999) 472.

[18] H.A. Dürr, S.S. Dhesi, E. Dudzik, D. Knabben, G. van der Laan, J.B. Goedkoop, F.U. Hillebrecht, Phys. Rev. B 59 (1999) R701.

[19] P. Gambardella, A. Dallmeyer, K. Maiti, M.C. Malagoli, W. Eberhardt, K. Kern, C. Carbone, Nature 416 (2002) 301.

[20] S.Y. Chou, IEEE Proc. 85 (1997) 652.

[21] D. Weller, A. Moser, IEEE Trans. Mag. 35 (1999) 4423.

[22] S. Sun, C.B. Murray, D. Weller, L. Folks, A. Moser, Science 287 (2000) 1989.

[23] H. Brune, Surf. Sci. Rep. 31 (1998) 121.

[24] O. Fruchart, M. Klaua, J. Barthel, J. Kirschner, Phys. Rev. Lett. 83 (1999) 2769.

[25] S.E. Apsel, J.W. Emmert, J. Deng, L.A. Bloomfield, Phys. Rev. Lett. 76 (1996) 1441-1444.

[26] J. Stöhr, J. Magn. Magn. Mater. 200 (1999) 470.

[27] B.T. Thole, P. Carra, F. Sette, G. van der Laan, Phys. Rev. Lett. 68 (1992) 1943-1946.

[28] P. Carra, B.T. Thole, M. Altarelli, X. Wang, Phys. Rev. Lett. 70 (1993) 694.

[29] C.T. Chen, Y.U. Idzerda, H.-J. Lin, N.V. Smith, G. Meigs, E. Chaban, G.H. Ho, E. Pellegrin, F. Sette, Phys. Rev. Lett. 75 (1995) 152.

[30] P. Gambardella, S. Rusponi, M. Veronese, S.S. Dhesi, C. Grazioli, A. Dallmeyer, I. Cabria, R. Zeller, P.H. Dederichs, K. Kern, C. Carbone, H. Brune, Science 300 (2003) 1130.

[31] P. Bruno, Phys. Rev. B 39 (1989) 865.

[32] G. van der Laan, J. Phys.: Condens. Matter. 10 (1998) 3239.

[33] I.V. Solovyev, P.H. Dederichs, I. Mertig, Phys. Rev. B 52 (1995) 13419.

[34] B. Lazarovits, L. Szunyogh, P. Weinberger, Phys. Rev. B 67 (2003) 024415.

[35] P. Gambardella, A. Dallmeyer, K. Maiti, M.C. Malagoli, S. Rusponi, P. Ohresser, W. Eberhardt, C. Carbone, K. Kern, Phys. Rev. Lett. 93 (2004) 077203.

[36] S. Rusponi, T. Cren, N. Weiss, M. Epple, P. Buluschek, L. Claude, H. Brune, Nat. Mater. 2 (2003) 546-551.

[37] W. Wernsdorfer, E. Bonet Orozco, K. Hasselbach, A. Benoit, B. Barbara, N. Demoncy, A. Loiseau, H. Pascard, D. Mailly, Phys. Rev. Lett. 78 (1997) 1791.

[38] W. Kinzel, K.H. Fischer, Sol. State Comm. 23 (1977) 687.

[39] R.W. Chantrell, N.Y. Ayoub, J. Popplewell, J. Magn. Magn. Mater. 53 (1985) 199.

[40] O. Fruchart, P.O. Jubert, C. Meyer, M. Klaua, J. Barthel, J. Kirschner, J. Magn. Magn. Mater. 239 (2002) 224.

[41] T. Koide, H. Miyauchi, J. Okamoto, T. Shidara, A. Fujimori, H. Fukutani, K. Amemiya, H. Takeshita, S. Yuasa, T. Katayama, Y. Suzuki, Phys. Rev. Lett. 87 (2001) 257201.

[42] R. Wu, C. Li, A.J. Freeman, J. Magn. Magn. Mater. 99 (1991) 71.

[43] S. Ferrer, J. Alvarez, E. Lundgren, X. Torrelles, P. Fajardo, F. Boscherini, Phys. Rev. B 56 (1997) 9848.

[44] J. Thiele, C. Boeglin, K. Hricovini, F. Chevrier, Phys. Rev. B 53 (1996) R11934.

[45] N.W.E. McGee, M.T. Johnson, J.J. de Vries, J. aan de Stegge, J. Appl. Phys. 73 (1993) 3418.

[46] A. Aharoni, J. Appl. Phys. 83 (1998) 3432.

[47] R.W. Chantrell, N. Walmsley, J. Gore, M. Maylin, Phys. Rev. B 63 (2001) 024410.

[48] C. Haginoya, S. Heike, M. Ishibashi, K. Nakamura, K. Koike, T. Yoshimura, Y. Yamamoto, Y. Hirayama, J. Appl. Phys. 85 (1999) 8327.

[49] M.P. Sharrock, IEEE Trans. Mag. 26 (1990) 193.

[50] J. García-Otero, A.J. García-Bastida, J. Rivas, J. Magn. Magn. Mater. 189 (1998) 377.

[51] M. Albrecht, T. Furubayashi, M. Przybylski, J. Korecki, U. Gradman, J. Magn. Magn. Mater. 113 (1992) 207.

[52] U. Gradmann, H.J. Elmers, T. Durkop, J. Magn. Magn. Mater. 165 (1997) 56.

[53] W. Weber, C.H. Back, A. Bischof, C. Würsch, R. Allenspach, Phys. Rev. Lett. 76 (1996) 1940.

[54] W. Weber, C.H. Back, A. Bischof, D. Pescia, R. Allenspach, Nature 374 (1995) 788.

[55] W. Weber, C.H. Back, U. Ramsperger, A. Vaterlaus, R. Allenspach, Phys. Rev. B 52 (1995) R14400.

[56] R.K. Kawakami, M.O. Bowen, H.J. Choi, E.J. Escorcia-Aparicio, Z.Q. Qiu, Phys. Rev. B 58 (1998) R5924.

[57] D.S. Chuang, C.A. Ballentine, R.C. O’Handley, Phys. Rev. B 49 (1994) 15084.

[58] P. Ohresser, N.B. Brookes, S. Padovani, F. Scheurer, H. Bulou, Phys. Rev. B 64 (2001) 104429.

[59] C.S. Shern, J.S. Tsay, H.Y. Her, Y.E. Wu, R.H. Chen, Surf. Sci. 429 (1999) L497.

[60] O. Robach, C. Quiros, P. Steadman, K.F. Peters, E. Lundgren, J. Alvarez, H. Isern, S. Ferrer, Phys. Rev. B 65 (2002) 054423.

[61] J.-W. Lee, J.-R. Jeong, S.-C. Shin, J. Kim, S.-K. Kim, Phys. Rev. B 66 (2002) 172409.

[62] J. Kim, J.-W. Lee, J.-R. Jeong, S.-C. Shin, Y.H. Ha, Y. Park, D.W. Moon, Phys. Rev. B 65 (2002) 104428.

[63] C.-J. Lin, G.L. Gorman, C.H. Lee, R.F.C. Farrow, E.E. Marinero, H.V. Do, H. Notarys, C.J. Chien, J. Magn. Magn. Mater. 93 (1991) 194.

[64] P.F. Carcia, A.D. Meinhaldt, A. Suna, Appl. Phys. Lett. 47 (1985) 178.

[65] B.N. Engel, M.H. Wiedmann, R.A. van Leeuwen, C.M. Falco, Phys. Rev. B 48 (1993) 9894-9897.

[66] J.-Y. Kim, B.-K. Lee, G.-S. Park, M. Uchida, T. Kurosawa, S. Watanabe, J. Ariake, N. Honda, K. Ouchi, J. Magn. Magn. Mater. 235 (2001) 53. 
[67] U. Pustogowa, J. Zabloudil, C. Uiberacker, C. Blaas, P. Weinberger, L. Szunyogh, C. Sommers, Phys. Rev. B 60 (1999) 414.

[68] J. Dorantes-Dávila, H. Dreyssé, G.M. Pastor, Phys. Rev. Lett. 91 (2003) 197206.

[69] J. Kohlhepp, U. Gradmann, J. Magn. Magn. Mater. 139 (1995) 347.

[70] C. Li, A.J. Freeman, H.J.F. Jansen, C.L. Fu, Phys. Rev. B 42 (1990) 5433.

[71] E. Lundgren, B. Stanka, M. Schmid, P. Varga, Phys. Rev. B 62 (2000) 2843.

[72] P. Beauvillain, A. Bounouh, C. Chappert, R. Mégy, S. Ould-Mahfoud, J.P. Renard, P. Veillet, D. Weller, J. Corno, J. Appl. Phys. 76 (1994) 6078.

[73] H. Fritzsche, J. Kohlhepp, U. Gradmann, J. Magn. Magn. Mater. 148 (1995) 154.

[74] T. Hill, M. Mozaffari-Sørensen Afshar, J. Schmidt, T. Risse, H.-J. Freund, Surf. Sci. 429 (1999) 264

[75] V. Skumryev, S. Soyanov, Y. Zhang, G. Hadjipanayis, D. Givord, J. Nogués, Nature 423 (2003) 850.

[76] T. Cren, S. Rusponi, N. Weiss, M. Epple, H. Brune, J. Phys. Chem. B 108 (2004) 14685.

[77] P. Gambardella, Z. Sljivancanin, B. Hammer, M. Blanc, K. Kuhnke, K. Kern, Phys. Rev. Lett. 87 (2001) 056103. 